\title{
An e-conversation with Prof. Jeffrey J. Siirola
}

\author{
Subhas K. Sikdar
}

Published online: 28 November 2014

(C) Springer-Verlag Berlin Heidelberg (Outside the USA) 2014

Subhas Sikdar Jeff, shortly after being elected to be the President of the American Institute of Chemical Engineers, you gave several key lectures on net global carbon dioxide emissions and the attendant global warming issues. I think this was a first for the President of this Institute. Global warming and its potential impact were being discussed frequently in the press and the professional journals at the time. In your lectures, you also suggested some mitigation options, not all practical, to illustrate the gravity of the challenge. Now that we know details of what the Intergovernmental Panel on Climate Change (IPCC) reported this year, what is your view of the various mitigation options to contain the global average temperature increase to, say $2{ }^{\circ} \mathrm{C}$ ?

Jeff Siirola Let me first say that I do not know what the relationship between atmospheric carbon dioxide concentration and global average temperature increase is, but then secondly that the severity of required mitigation options may not depend that much on any specific degree of temperature increase or atmospheric concentration. Let me elaborate.

We happen to have fairly direct evidence from Antarctic and Greenland ice cores of atmospheric carbon dioxide (and methane) concentrations and average global temperatures over the last 650,000 years covering eight glacial periods. The carbon dioxide concentrations varied from about $180 \mathrm{ppm}$ (glacial period) to about $280 \mathrm{ppm}$ (interglacial period), while the average temperature varied by about $12 \mathrm{~K}$. The most obvious conclusion drawn from the data is that they are correlated. Cause and effect are less

S. K. Sikdar $(\bowtie)$

Cincinnati, USA

e-mail: sikdar.subhas@epa.gov clear. Close examination of the data indicates that the temperature signal leads the carbon dioxide response by about 600-800 years. This would imply that temperature change, likely due to solar insolation changes resulting from variations in Earth's axial precession, axial tilt, and orbital eccentricity as proposed by Milanković a century ago, was the independent cause and that the dependent carbon dioxide response was a result of ocean degassing (decreased surface solubility at warmer temperatures). Although the average surface temperature is in fact influenced by greenhouse mechanisms due to water, carbon dioxide, methane, and other atmospheric components, it would appear solar forcing, not atmospheric composition, played the greatest role in temperature determinations in that era, since, for example, some of the most rapid decreases in temperature occurred in periods of the highest carbon dioxide concentration.

The last glacial (cold) period ended about 15,000 years ago. We are now near the peak of the subsequent interglacial period. For the last $2.6 \mathrm{M}$ years, even in the interglacial periods, Arctic, Antarctic, and Greenland ice sheets have remained. Before the beginning of the industrial revolution, the atmospheric carbon dioxide concentration was $280 \mathrm{ppm}$ (about the same as peak concentrations in other interglacial periods), and the average temperature may have already peaked and started a subsequent decrease. Sea levels also changed by about $125 \mathrm{~m}$ between glacial and interglacial periods. There is evidence that current sea level is about $6 \mathrm{~m}$ lower than the maximum level attained in the previous interglacial period 125,000 years ago. The current level has been nearly constant for the last 6,000 years and may very well have peaked for the current cycle. However, it is clear that neither sea levels nor the positions of the shoreline are constant. 
Now, since the industrial revolution and the large scale and increasing combustion of fossil fuels, large additional amounts of carbon dioxide are being emitted to the atmosphere, currently at a rate exceeding 7GTC (gigatons carbon) per year. It is believed about $2.5 \mathrm{GTC} /$ year of carbon is dissolving in the ocean resulting in $4.5 \mathrm{GTC} /$ year net addition to the atmosphere. The atmospheric inventory is about 750GTC, so these additions have brought the current atmospheric carbon dioxide concentration to $400 \mathrm{ppm}$, currently increasing at 2 ppm per year.

Human-caused carbon dioxide increase has now exceeded the magnitude of glacial period carbon dioxide concentration variation, and there is widespread concern that even if carbon dioxide (or other gas) increases did not "cause" glacial period temperature changes, and that eventually by greenhouse mechanisms (molecular absorption interference with nighttime infrared radiation into space), the global average temperatures will increase for reasons other than solar insolation variation, with eventual impacts including additional glacier melt, rising sea levels, more intense weather events, shifting rainfall and agriculture patterns, and other climate change indicators.

Instrumental evidence shows that since the beginning of the industrial revolution, global average temperature has increased by $0.8 \mathrm{~K}$. The data has short term variability which seems correlated with the 11-year sun spot cycle, but the long-term increasing trend is clear. This is not a proof that the increase has been caused by human carbon dioxide emissions, or that the observed temperature increase is what should be expected from a carbon dioxide concentration increase of $120 \mathrm{ppm}$, or that if the atmospheric concentrations were frozen at the current $400 \mathrm{ppm}$ level, how much additional temperature rise if any would occur, but to many people the present situation is a cause for serious concern.

I do not have the expertise to speculate on how much average temperature change is "acceptable," and what the costs (and benefits) of any particular change might be, although I understand there are increasing efforts to view climate change impacts and mitigation from an economic risk management point of view. So, I do not know what an acceptable global average temperature is, and I do not know what concentration of absorbing gases in the atmosphere is consistent with that temperature at steady state. But to get to the second part of my answer, the cost of achievement may not much depend on either parameter (except if society decides to significantly decrease an atmospheric contaminant concentration already present).

If society in its collective wisdom decides on the basis of impacts, costs, data, models, and other factors that atmospheric concentration of contaminants such as carbon dioxide (and methane and others) should be limited, then this implies that all mechanisms adding the contaminants to the atmosphere must be balanced (over some reference time) by all mechanisms, by removing the contaminant from the atmosphere. Otherwise, the accumulation term will be nonzero, and the concentration will not be limited.

For carbon dioxide, at the present time, the principal sources of gas to the atmosphere are biological decay and fossil fuel combustion. The principal sinks of gas from the atmosphere are biological photosynthesis and oceanic absorption. Global biological photosynthesis and biological decay are about in balance (both about 60GTC/year), with some local imbalances because of not only deforestation but also reforestation. Oceanic absorption involves many mechanisms including photosynthetic production of biological materials which ultimately sink avoiding oxidative decomposition (the biological pump), and inorganic mechanisms increasing the bicarbonate ion concentration of the ocean (and decreasing its $\mathrm{pH}$ ). As mentioned before, the present net oceanic absorptions are believed to remove 2.5GTC/year of new carbon dioxide concentration from the atmosphere. The various mechanisms are complex. The rates of some may be a function of atmospheric carbon dioxide concentration, and these rates may increase as atmospheric carbon dioxide concentrations increase. Most mechanisms, however, depend on details of circulation below the ocean surface.

So, here is the conundrum. The current carbon dioxide emissions to the atmosphere are on the order of 7GTC/year. Some estimates are within a half century or so; if populations and standards of living increase, these emissions would grow to on the order of 30GTC/year. And yet, the oceanic removal rate is but $2.5 \mathrm{GTC} / \mathrm{year}$. If the atmospheric concentration of carbon dioxide is to be stabilized (at whatever level, it does not matter except to the extent some oceanic absorption mechanisms may be atmospheric concentration dependent), then net atmospheric emissions must be reduced to the same level of $2.5 \mathrm{GTC} /$ year. This means a reduction of $65 \%$ from today's emissions and potentially more than $90 \%$ from future emissions. Again, this amount of mitigation is (nearly) independent of the atmospheric concentration or the average global temperature.

These reductions could come from carbon capture and sequestration, and by not emitting in the first place (providing energy for the needs of society by methods not involving fossil fuel combustion and carbon emission). The balance might also be maintained if mechanisms were invented to increase the rate of oceanic absorption, although the deleterious consequences of the decreasing $\mathrm{pH}$ must also be mitigated (as by carbonate neutralization). Several decades of research have been done on both of the first two options. Costs are high, and to a first approximation would be about double the cost of electricity, but this is not necessarily prohibitive as some have argued. And it is because these costs are within the realm of 
possibility that some have proposed mechanisms such as a properly structured carbon tax to encourage innovation and action.

While there are no commercial examples of capture and sequestration, there are limited examples of non-fossil wind and photovoltaic electricity generation (under subsidies, but with other issues because of the nondispatchable character of these time-variant sources), and even more examples of fuel switching (natural gas for coal) by utilities and large industries, encouraged by the threat of taxes and the new abundance of relatively inexpensive shale gas. Some foresee the electrification of transportation and domestic heating facilitated by very efficient gas-combined cycle generation (eventually with carbon capture) as a further way to reduce carbon emissions in these sectors (made on the grounds of economic and energetic efficiency as well as carbon mitigation).

Economists can calculate a more definitive number. In my industrial and personal experience, energy seems to be on the order of $10 \%$ of expenditures. Doubling this cost in order to prevent adverse climate change is likely to be much less than the costs of adapting to the adverse consequences of climate changes that would never stabilize.

SKS Very well. It appears from your back of the envelope calculation, and from policy suggestions by the pundits, both the options, mitigation and adaptation, will cost a lot of money. And from a realistic policy point of view, we would do not just one, but we would do both, compounding the cost. Four sustainability questions appear here: (1) carbon tax on all carbon, and adaptation cost, will be disproportionately borne by the poor, creating social unrest. We are now talking about possibly accepting longer-term sustainability - if it works at all, while creating a shorterterm problem; (2) Second, if this is not done worldwide, would it not lead to the unintended global outcome? As Kyoto, Durban, and Copenhagen have demonstrated, this might be wishful thinking; (3) If the first two scenarios look bleak, is there an interim approach, in your opinion, which can do some good, giving us enough time to verify the possible causal connection between man-caused GHG and average global temperature, based on which, other options can be considered? and (4) can nuclear energy not have a role, by way of say, breeder reactor, thorium power, and eventually fusion?

JJS While it is true that the costs of many essential services-food, shelter, transportation, energy, public safety, and yes, environmental impact mitigation-are disproportionately borne by the less affluent because of the use of such services as a proportion of income, the service costs are nevertheless largely proportional to use. There are a plenty of existing and potential policy schemes that can be instituted to relieve such burdens, so that such service costs in general and carbon management costs in particular need not lead to particular social unrest. Furthermore, anecdotal evidence suggests that the potential for such social disturbance-if carbon taxes were instituted, for example, at a rate that would double the cost of electricity-may be overestimated as, for a variety of reasons, the retail price of electricity has already varied by more than a factor of two in different parts of the country without significant public outcry.

However, the issue of imposing usage constraints, emission caps, or fees and taxes in a non-uniform manner across the world is real and serious. Even if carbon mitigation "only" doubled the cost of energy and even if energy were only about ten percent of typical expenses (manufacturing costs, family budgets, etc.), an increase of ten percent in costs in one country but not another is competitively disastrous and economically fatal as such an amount may exceed profit margins. And as you point out, previous attempts at global agreements as at Kyoto, Durban, and Copenhagen were not successful. It is my opinion that the failure of those global agreement attempts was also used as an excuse by many companies to delay or cancel investments in carbon management including support for research and technology development on the grounds of mortal non-competitiveness in the absence of universal requirements. I believe that it has been a convenient excuse for inaction. However, I remain a bit optimistic. I actually think it could be possible to negotiate some kind of agreement among the major generator nations. I do not know exactly what the details of such an agreement might be, but I expect that it would have to have certain features such that all parties perceived it as fair to them and that compliance by all could be readily verified. I suspect that details such as at what rate emissions were to be decreased or atmospheric concentrations stabilized or lowered or the mechanisms by which that would be achieved would be far less important than the transparency and the assurance that no party could cheat. That would eliminate the current incentives to delay action, as then those quickest in achieving compliance could instead become the most economically advantaged. I would like to see far more effort devoted to exploring the construction of frameworks for such agreements that could be acceptable at least initially by the major emitting nations.

Although the exact amounts differ by geographic region, approximately one-half of carbon emissions come from the combustion of petroleum (mostly the transportation sector), about one-third from the combustion of coal (mostly the electric power generation sector), and about one-sixth from the combustion of natural gas (until recently, mostly industrial and residential heating). In the immediate term, especially given the increased availability, the decreased 
cost of shale natural gas, and the relative ease of substituting gas for coal in boilers (albeit with some power derating), it would appear that fuel-switching will be an initial, economic, and low-capital-cost strategy to decrease carbon generation by on the order of $20 \%$ in the United States and some other parts of the world. This, of course, is already well underway and has led to a $25 \%$ increase in natural gas consumption in the U.S. in less than a decade (after being approximately constant for the previous three decades). I believe this trend will continue until almost all coal consumption in boilers is replaced in regions where inexpensive methane is available.

In the next time period, I believe much of the new installed electric power production capacity will use natural gas-combined cycle technologies almost doubling electricity generation efficiency compared with the Rankine cycle facilities they will replace. Not only does this further decrease carbon generation, but the additional electricity generation capacity could support electrification of increasing portions of the transportation sector, including trains and many urban vehicles, and the building heating sectors (by way of heat pump technology). The initial benefits are higher system thermodynamic efficiency and lower carbon emissions compared with internal combustion engines and direct space heating. Also, increased electrification of transportation and industrial and residential heating later facilitates centralized carbon capture from the generation facilities, when those technologies are eventually developed and implemented compared with the impracticality of carbon capture from the current mobile transportation and distributed residential sectors.

Another benefit of the increasing electrification of various sectors of the economy is the facilitation of non-carbonaceous methods of electricity generation including hydroelectric (static, kinetic, and tidal), photovoltaic, wind, geothermal, and nuclear. Although some of these sources have been proposed as energy sources in other forms especially thermal, technologies are already well known to exploit these sources as electricity, and these methods, coupled with the increasing proportion of electricity-driven end-uses, directly reduce carbon generation. All of these non-carbonaceous methods have various environmental, technical, economic, dispatchability, or social acceptance challenges. However, all including new nuclear fuel cycles, fast reactors, spent-fuel reprocessing, waste transmutation, and other strategies can contribute to the accelerated transition to reduced greenhouse gas production and deserve serious continued development and evaluation.

SKS Thanks. At this point, I want to change the focus to pick your brain on some other issues I am curious about. You spent all of your pre-academic life in industry doing process design, development, simulation, and optimization and related tasks. You have had a significance presence in computer-aided process engineering. Undoubtedly, these developments on aggregate have tremendously elevated our ability to design and manage processes for chemical and petrochemical industries. I would like to know your opinion on where we stand today. What are the current challenges in this field especially with respect to resource conservation, recycle, and reuse? Are these computer-aided techniques penetrating processes outside of chemical industry?

JJS I look at chemical process design as a series of interconnected formulation-synthesis-specification-analysis-evaluation-optimization-control tasks in the areas of underlying science (chemistry, physics, and increasingly biology), governing phenomena, process equipment, manufacturing plant, firm, and supply chain. These areas have different scales, and the design tasks are generally performed iteratively at increasing levels of detail (including, e.g., targeting, conceptual, preliminary, definitive, and detailed). Digital computation has facilitated tremendous design capability advances-first in analysis, and then in optimization, and control in most process systems areas and levels of detail—and is beginning to have an impact in the synthesis of alternatives tasks as well.

There is increased interest in the concept of process intensification which might be defined as design modifications which result in systems that meet all the specified process goals and constraints, but which do so while simultaneously improving performance of some additional objectives which could include inherent safety, capital or operating costs, energy requirements, raw material consumption, employment, environmental impacts, flexibility, robustness, reliability, controllability, physical size, esthetics, life cycle considerations, and the like. The resource conservation, recycle, and reuse challenges you mention are indeed examples of such objectives. In some cases, some of these additional objectives can be monetized and combined into a single overall economic objective function. In other cases, it is desired to consider some of these objectives separately leading to multi-objective optimization and the analysis of Pareto-optimal alternatives. Over 40 aspects of process intensification have been categorized, some which have also been identified as the principles of Green Chemistry and Green Engineering, ranging from simple heat and power integration to reaction selectivity-improvement techniques to enhanced heat, and mass-transfer devices to inherently safer practices. Awareness of these principles during the design phase is increasing, and application is more limited by imagination and time than by lack of analysis, evaluation, or 
optimization tools. Similar principles_-for use once a plant is built, for example, Smart Manufacturing (the application of massive numbers of sensors and real-time computational resources in support of maximizing many of these additional objectives in addition to fitness-for-use goals and constraints during operations)_are at an earlier stage of development, and their ability to deliver the magnitude of results as promised are yet to be demonstrated. Also, some process intensification techniques, like wide use of heat integration, while improving energy consumption objectives, may simultaneously worsen other objectives such as robustness, flexibility, and controllability. The ability of operational techniques like Smart Manufacturing to possibly offset or reverse such adverse consequences may actually prove to be a major enabler to the increased application of process intensification and similar techniques directed toward improved environmental and life cycle objectives.

SKS Your idea of process design is indeed a healthy and useful insight. This much broader view always keep continual innovation in focus. Continual improvement is essentially the basis of sustainability concept in process design. As you say, there are many desirable objectives before us, and some may not yet be known. Thus, even when the techniques and tools may be available, we are handicapped by our limited imagination of how to define all the sub-objectives of a design. For various practical reasons, the best course of action then is incremental but continual improvement. Your observation of smart manufacturing to tackle the tradeoffs in process intensification is very insightful as well.

Now I want to move to engineering education as a topic. Even when you were in industry, you had close ties to academia, and had served on ABET committees. It would be interesting to know your thoughts on how engineering education is doing in this country. And, particularly, are we as a country producing enough engineers? The picture of the graduate schools clearly point to the negative. Are the STEM programs having much influence?

JJS For the last several years since my retirement from industry, I have been able to teach both undergraduate and graduate courses at two universities. In many ways, the undergraduate chemical engineering curriculum has not changed very much in the last 40 years since the wide adoption of 'chemical engineering science' including particularly transport phenomena and modern reaction engineering. The exception is the much increased use of digital computation especially in not only process simulation but also in process control, computational fluid dynamics, and similar modeling applications. There is also the increased emphasis in biology as both an underlying process science and as an area of application, as well as in emerging areas such as product design, process synthesis, and optimization.

The students I have encountered are as strong in the fundamentals as previous generations, although fewer have computer programming skills (which anecdotally have correlated with process design creativity), and fewer have had industrial co-op or intern experiences (and the practical insights they bring) opting instead for undergraduate laboratory research and international study. Nevertheless, largely because of the increased use of computational tools especially simulators, the quality of student work, for example, in the capstone process design courses I mentor, is much better than that produced by earlier generations by hand and approximate method, and that of course is very welcome.

I can only speak for chemical engineering. We have over 150 chemical engineering programs in the country. They are not all the same, and they are not supposed to be. Each has different constituents, different program objectives, different curricula, and different instructional methods. But it is my observation through several decades of accreditation activities, service on advisory boards, and now through mentoring at two institutions, that the capabilities of those now entering the profession are very high. Of course needs are always increasing, so there will always be a requirement for life-long learning as well as continuous improvements in the undergraduate experience.

As for the number of engineers, my understanding is that, even as the country continues to recover from the recent recession, the graduates are readily finding jobs within their fields of expertise; that unemployment among engineers is very low; and that in many sectors, needs are being met with additional international workers. That would certainly imply that there is not an oversupply of engineers. Many institutions are setting increased engineering and other STEM field enrollment goals and are putting in place additional faculty and infrastructure to accommodate these increased goals. Looking at expected growth, domestically because of long-term advantaged energy supplies, and internationally because of widespread improved economic conditions, and also because of increasing interests in adequate and high-quality food and water, environmental impact mitigation including climate change, and many other sustainability goals, I would surmise that the increasing needs for engineers and those with related expertise will not abate any time soon. There may always be short-term or local aberrations, but I believe the future to be brighter than any time in the past. It really is a very exciting time to be in our profession. 
SKS Thank you very much, Jeff, for sharing your ideas on technical matters of interest to this journal, which are also of great significance to the profession of engineering and to the society at large. It was a pleasure conversing with you.

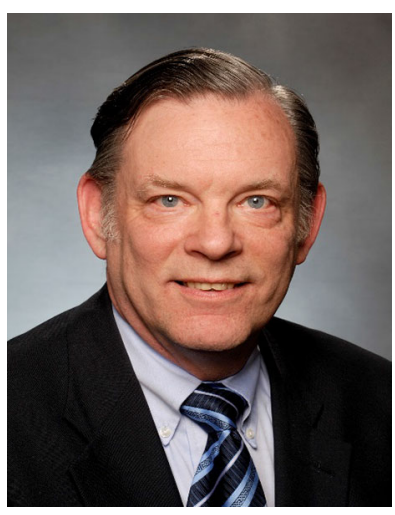

Jeff Siirola retired in 2011 as a Technology Fellow at the Eastman Chemical Company in Kingsport Tennessee where he had been for more than 39 years. He now holds half-time positions as Professor of Engineering Practice at Purdue University and Distinguished Service Professor of Sustainable Energy Systems at Carnegie Mellon University. Siirola received a $\mathrm{PhD}$ in chemical engineering from the University of Wisconsin-Madison in 1970. His areas of interest include chemical process synthesis, computer-aided conceptual process engineering, design theory and methodology, chemical process development and technology assessment, resource conservation and recovery, sustainable development and growth, carbon management, and chemical engineering education. Siirola is a member of the National Academy of Engineering and was in 2005 the President of the American Institute of Chemical Engineers. 